\title{
Recursive Integral Terminal Sliding Mode Control of MEMS Gyroscopes via Composite Neural Learning and Disturbance Observer
}

Rui Zhang ( $\square$ ruizhangdyx@hotmail.com )

Southeast University https://orcid.org/0000-0002-4554-7718

Bin Xu

Northwestern Polytechnical University

Hai Wang

Murdoch University

\section{Research Article}

Keywords: gyroscope, recursive integral terminal sliding mode control, serial-parallel estimation model, composite neural learning, disturbance observer.

Posted Date: January 24th, 2022

DOI: https://doi.org/10.21203/rs.3.rs-1282195/v1

License: (c) (i) This work is licensed under a Creative Commons Attribution 4.0 International License.

Read Full License 


\title{
Recursive Integral Terminal Sliding Mode Control of MEMS Gyroscopes via Composite Neural Learning and Disturbance Observer
}

\author{
Rui Zhang · Bin Xu · Hai Wang
}

Received: date / Accepted: date

\begin{abstract}
The recursive integral terminal sliding mode control based on disturbance observer and composite neural learning is studied in this paper to control the dynamics of MEMS gyroscopes in the presence of system uncertainties and external disturbances. To achieve more accurate approximation accuracy of system uncertainties, the composite neural learning is employed, where the updating law of the weight vector is designed by the tracking error and the prediction error constructed by serial-parallel estimation model. To further improve the system robustness, the recursive integral terminal sliding mode controller is utilized, where faster convergence can be obtained by forcing the system state to start from the sliding mode manifold at the initial time. To deal with external disturbances with unknown upper bounds, the disturbance observer is designed. Furthermore, simulations results are demonstrated to verify that faster convergence and higher tracking accuracy can be achieved under the proposed method.
\end{abstract}

Keywords MEMS gyroscope, recursive integral terminal sliding mode control, serial-parallel estimation model, composite neural learning, disturbance observer.

\section{R. Zhang (ه)}

School of Automation, Southeast University, Nanjing 210096, China

E-mail: ruizhangdyx@ @otmail.com

B. Xu

School of Automation, Northwestern Polytechnical University, Xi' an 710072, China

H. Wang

Discipline of Engineering and Energy, Murdoch University, Perth, WA 6150, Australia

Centre of Water, Energy \& Waste, Harry Butler Institute, Murdoch University, Perth, WA6150, Australia

\section{Introduction}

The gyroscopes using micro electro mechanical systems (MEMS) technology are widely utilized to measure the angular velocity in various fields such as electronics, robots, aeronautics and astronautics. There are two control frames for MEMS gyroscopes: the conventional "one axis drive and one axis detection" control frame using weak signal detection technology to detect the angular velocity, and the "biaxial drive" control frame [1] using an adaptive law to estimate the angular velocity online. Since weak signal detection technology is difficult to be further improved, the detection limit of angular velocity under the first control frame can not be broken. In response to this problem, advanced control methods under the "biaxial drive" control frame have received more attention.

Under the "biaxial drive" control frame, the proportionalintegral (PI) control is widely used in practical applications. However, due to the inevitable existence of system uncertainties and external disturbances in the dynamics of MEMS gyroscopes, the tracking accuracy of PI control is reduced. For the system uncertainties, the adaptive method is introduced in [2] to estimate the uncertainties, which is further fed to the controller for compensation. Owing to the ability of radial basis function (RBF) neural networks (NN) for the approximation of every nonlinear function with any accuracy $[3,4]$, the RBF NN is employed to approximate system uncertainties in $[5,6]$. To further improve the approximation performance of NN, finite time learning [7] and multiloop recurrent NN [8] are proposed. For the external disturbances, when the upper bounds of the disturbances are known, a robust controller is designed in $[9,10]$ to suppress the influence of disturbances. When the upper bounds of the disturbances exist but are unknown, the disturbance observer (DOB) based on Lyapunov method is designed in [11-13]. Furthermore, the active disturbance rejection control is pro- 
posed in $[14,15]$, where the lumped disturbances including system uncertainties and external disturbances are set as a new state and further introduced to the extended state observers, such that the lumped disturbances are accurately estimated. To improve the tracking accuracy of control system in the applications, more advanced intelligent control methods and disturbance observation control theories need to be studied.

Considering the second order dynamic model of MEMS gyroscopes, the back-stepping control scheme is applied to control MEMS gyroscopes in [16], where the second order dynamics can be decomposed into two first order subsystem$\mathrm{s}$ and the virtual controllers are proposed step by step. To avoid the parameters expansion, the dynamic surface controllers are further studied in $[17,18]$. However, the layered design of back-stepping control is too complex. Since sliding mode variable is able to contain the errors of differen$\mathrm{t}$ orders, it is introduced to the control system of MEMS gyroscopes in $[19,20]$. Following with that, various modified sliding mode control (SMC) are studied. The terminal SMC (TSMC) [21,7] and the super-twisting SMC [22] are addressed to force the sliding mode variable to achieve finite time convergence. In [23,24], the nonsingular TSMC is discussed to avoid the singular problem of conventional TSMC. In addition, various intelligent control methods and disturbance observation control theories are combined with advanced modified SMC to improve the tracking performance of MEMS gyroscopes with system uncertainties and external disturbances. Overall, the improvement of robustness and convergence is the key issue of SMC for MEM$\mathrm{S}$ gyroscopes, which will continue to receive attention in the future research.

Motivated by the above studies on the controller design of MEMS gyroscopes, this paper places emphasis on how to efficiently approximate the compound nonlinearity and how to improve the convergence rate of control system. A high precision and robust control scheme is designed to control the MEMS gyroscopes. The recursive integral terminal sliding mode controller (RTSMC) is utilized to achieve faster convergence and higher tracking accuracy, while the composite neural learning and DOB are designed to handle the system uncertainties and external disturbances. Compared with previous works, the main contributions are listed as follows.

- The RTSMC is designed to control the dynamics of MEM$S$ gyroscopes in the presence of system uncertainties and external disturbances. When the control system is forced to start from the sliding surface at the initial time, faster convergence and higher tracking accuracy can be obtained.

- The composite neural learning works together with the DOB to approximate the lumped uncertainty including system uncertainties and external disturbances. Through updating the NN weight vector by the adaptive law, which is designed by the tracking error and the prediction error constructed by the serial-parallel estimation model (SPEM), higher approximation accuracy is obtained.

The rest of this paper is organized as follows. The dynamics of MEMS gyroscopes and problem formulation are addressed in Section 2. The RTSMC using composite neural learning and DOB is designed in Section 3. The system stability analysis is demonstrated in Section 4. The simulation results are shown in Section 5 to verify the expected faster convergence and higher tracking accuracy. The conclusions are given in Section 6.

\section{Problem Formulation}

\subsection{MEMS Gyroscopes Dynamics}

MEMS gyroscopes are essentially mechanical gyroscopes, whose dynamics can be described as a mass-stiffness-damping system shown in Fig.1, where $x$ denotes the drive axis, $y$ denotes the sensitive axis, $\vartheta_{x}$ and $\vartheta_{y}$ denote dimensionless positions, $\varsigma_{x x}$ and $\varsigma_{y y}$ denote dimensionless damping coefficients, $\psi_{x x}$ and $\psi_{y y}$ denote dimensionless stiffness coefficients, $\psi_{x 3}$ and $\psi_{y 3}$ denote dimensionless nonlinear coefficients.

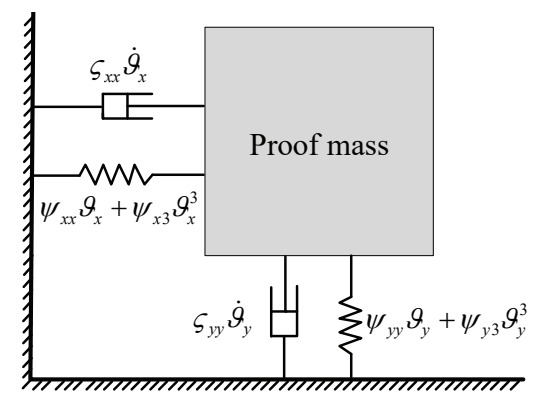

Fig. 1 Structure design of MEMS gyroscopes.

Considering the parameters uncertainties and the external disturbances, the dimensionless dynamics of MEMS gyroscopes with the mechanical coupling caused by machining errors is given as follows [25]

$\ddot{\vartheta}_{i}=f_{i}+d_{i}+\tau_{i}, i=x, y$

with

$$
\begin{aligned}
f_{x}= & -\varsigma_{x x} \dot{\vartheta}_{x}-\left(\varsigma_{x y}-2 \Omega\right) \dot{\vartheta}_{y}-\left(\psi_{x x}-\Omega^{2}\right) \vartheta_{x} \\
& -\psi_{x y} \vartheta_{y}-\psi_{x 3} \vartheta_{x}^{3} \\
f_{y}= & -\varsigma_{y y} \dot{\vartheta}_{y}-\left(\varsigma_{y x}+2 \Omega\right) \dot{\vartheta}_{x}-\left(\psi_{y y}-\Omega^{2}\right) \vartheta_{y} \\
& -\psi_{y x} \vartheta_{x}-\psi_{y 3} \vartheta_{y}^{3}
\end{aligned}
$$

where $\varsigma_{x y}$ and $\varsigma_{y x}$ denote dimensionless damping coupling coefficients, $\psi_{x y}$ and $\psi_{y x}$ denote dimensionless stiffness coupling coefficients, $d_{x}$ and $d_{y}$ denote dimensionless external 
disturbances, $\tau_{x}$ and $\tau_{y}$ denote dimensionless control inputs, $\Omega$ denotes the dimensionless angular velocity.

Defining the dimensionless reference signal as $\vartheta_{\text {ri }}$, the tracking error is given as

$e_{i}=\vartheta_{i}-\vartheta_{r i}$

Assumption 1 To avoid the mechanical damage, the external disturbance is assumed to be bounded. In other words, there exists constants $d_{m i}>0$ and $\bar{d}_{m i}>0$, such that $\left|d_{i}\right| \leq$ $d_{m i},\left|\dot{d}_{i}\right| \leq \bar{d}_{m i}$.

Remark 1 Considering the parameters uncertainties caused by environmental changes such as temperature, atmospheric pressure, etc., there are $\varsigma_{l_{1} l_{2}}=\varsigma_{l_{1} l_{2}}^{0}+\Delta \varsigma_{l_{1} l_{2}}, \psi_{l_{1} l_{2}}=\psi_{l_{1} l_{2}}^{0}+$ $\Delta \psi_{l_{1} l_{2}}$ and $\psi_{l_{1} 3}=\psi_{l_{1} 3}^{0}+\Delta \psi_{l_{1} 3}$ with $l_{1}=x, y$ and $l_{2}=x, y$, where $\varsigma_{l_{1} l_{2}}^{0}, \psi_{l_{1} l_{2}}^{0}$ and $\psi_{l_{1} 3}^{0}$ are nominal terms, $\Delta \varsigma_{l_{1} l_{2}}, \Delta \psi_{l_{1} l_{2}}$ and $\Delta \psi_{l_{1} 3}$ are uncertain terms.

\subsection{Composite Neural Learning}

Since the parameters of dynamics are changing with environmental factors such as temperature, atmospheric pressure, etc., the nonlinear dynamics $f_{i}$ is constantly changing. Considering the RBF NN can approximate every nonlinear function with any accuracy [26], it is employed to approximate $f_{i}$ as

$f_{i}\left(\zeta_{\text {in }}\right)=\varpi_{i}^{T} \theta\left(\zeta_{\text {in }}, \eta, \kappa\right)+\varepsilon_{i}$

where $\zeta_{\text {in }}=\left[\vartheta_{x}, \vartheta_{y}, \dot{\vartheta}_{x}, \dot{\vartheta}_{y}\right]^{T} \in \mathfrak{R}^{4 \times 1}$ is the input vector, $\varpi_{i} \in \mathfrak{R}^{n \times 1}$ is the optimal weight vector, $\theta(\cdot) \in \mathfrak{R}^{n \times 1}$ is basis function vector with $\eta \in \mathfrak{R}^{4 \times 1}$ as the center and $\kappa$ as the width, $\varepsilon_{i}$ is the approximation error with $\left|\varepsilon_{i}\right| \leq \varepsilon_{m}$ and $\varepsilon_{m}>0$.

From the dynamics (1), the SPEM is constructed as

$\ddot{\hat{\vartheta}}_{i}=\hat{\omega}_{i}^{T} \theta+d_{i}+\tau_{i}+c_{i} z_{i}$

where $\hat{\varpi}_{i}$ is the estimation of $\bar{\varpi}_{i}, c_{i}>0$ is an user-defined constant, $z_{i}$ is the prediction error implying the approximation error which is constructed as

$z_{i}=\dot{\vartheta}_{i}-\dot{\hat{\vartheta}}_{i}$

where $\dot{\hat{\vartheta}}_{i}$ is the estimation of $\dot{\vartheta}_{i}$.

The updating law of $\hat{\boldsymbol{\omega}}_{i}$ is designed as

$\dot{\hat{\sigma}}_{i}=\left(\varsigma_{1 i} e_{i}+\varsigma_{2 i} z_{i}\right) \theta-\imath_{i} \hat{\bar{\omega}}_{i}$

where $\varsigma_{1 i}>0, \varsigma_{2 i}>0$ and $\boldsymbol{l}_{i}>0$ are user-defined constants.

Remark 2 The conventional updating law of NN weight is designed as $\dot{\hat{\sigma}}_{i}=\varsigma_{1 i} e_{i} \theta-\imath_{i} \hat{\bar{\omega}}_{i}$. It is observed that only the tracking error $e_{i}$ is utilized to update $\hat{\omega}_{i}$, where the approximation error $\varepsilon_{i}$ is ignored. Therefore, the approximation performance of NN cannot be evaluated. In response to this problem, the SPEM-based prediction error is constructed, where $\varepsilon_{i}$ is implied in $z_{i}$. Hence, the approximation accuracy can be improved by designing the updating law (7) using $e_{i}$ and $z_{i}$.

\subsection{Control Goal}

The goal is to design a high precision and robust control scheme such that the system state can be forced to follow the dimensionless reference trajectory $\vartheta_{r i}$ when system uncertainties caused by environmental factors and external disturbances exist. Under the proposed controller, faster convergence and higher tracking accuracy of system tracking are expected.

\section{Controller Design}

For the dimensionless dynamics of MEMS gyroscopes (1), the RTSMC is designed to obtain higher tracking accuracy, while faster convergence is achieved by forcing the control system to start from the sliding surface at the initial time.

Considering the nonlinear dynamics including system uncertainties, RBF NN is adopted in this work. Differen$\mathrm{t}$ from the previous works where the updating law is constructed by the tracking error [5,6], the updating law of composite neural learning is designed by the tracking error and the prediction error constructed by SPEM. To deal with the external disturbances, DOB is further developed. The diagram of the control scheme is presented in Fig. 2.

The recursive integral terminal sliding mode variable [2729] is constructed as

$s_{i}=\chi_{i}+\rho_{i} \chi_{i}^{I}$

with

$\chi_{i}=\dot{e}_{i}+\ell_{i} e_{i}+\lambda_{i}\left|e_{i}\right|^{\alpha_{i}} \operatorname{sign}\left(e_{i}\right)$

$\dot{\chi}_{i}^{I}=\left|\chi_{i}\right|^{\beta_{i}} \operatorname{sign}\left(\chi_{i}\right)$

where $\alpha_{i}>1,0<\beta_{i}<1, \rho_{i}>0, \ell_{i}>0$ and $\lambda_{i}>0$ are userdefined constants.

The derivative of $s_{i}$ is calculated as

$$
\begin{aligned}
\dot{s}_{i}= & \dot{\chi}_{i}+\rho_{i} \dot{\chi}_{i}^{I} \\
= & \ddot{e}_{i}+\ell_{i} \dot{e}_{i}+\alpha_{i} \lambda_{i}\left|e_{i}\right|^{\alpha_{i}-1} \dot{e}_{i}+\rho_{i} \dot{\chi}_{i}^{I} \\
= & \ddot{\vartheta}_{i}-\ddot{\vartheta}_{r i}+\ell_{i} \dot{e}_{i}+\alpha_{i} \lambda_{i}\left|e_{i}\right|^{\alpha_{i}-1} \dot{e}_{i}+\rho_{i} \dot{\chi}_{i}^{I} \\
= & \varpi_{i}^{T} \theta\left(\zeta_{i n}, \eta, \kappa\right)+\varepsilon_{i}+d_{i}+\tau_{i}-\ddot{\vartheta}_{r i} \\
& +\ell_{i} \dot{e}_{i}+\alpha_{i} \lambda_{i}\left|e_{i}\right|^{\alpha_{i}-1} \dot{e}_{i}+\rho_{i} \dot{\chi}_{i}^{I}
\end{aligned}
$$

The controller is designed as

$\tau_{i}=\tau_{a i}+\tau_{d i}+\tau_{n i}+\tau_{s i}$

with

$$
\begin{aligned}
\tau_{a i} & =-\hat{\varpi}_{i}^{T} \theta \\
\tau_{d i} & =-\hat{D}_{i} \\
\tau_{n i} & =\ddot{\vartheta}_{r i}-\ell_{i} \dot{e}_{i}-\alpha_{i} \lambda_{i}\left|e_{i}\right|^{\alpha_{i}-1} \dot{e}_{i}-\rho_{i} \dot{\chi}_{i}^{I} \\
\tau_{s i} & =-\mu_{1 i} s_{i}-\mu_{2 i}\left|s_{i}\right|^{v_{i}} \operatorname{sign}\left(s_{i}\right)
\end{aligned}
$$




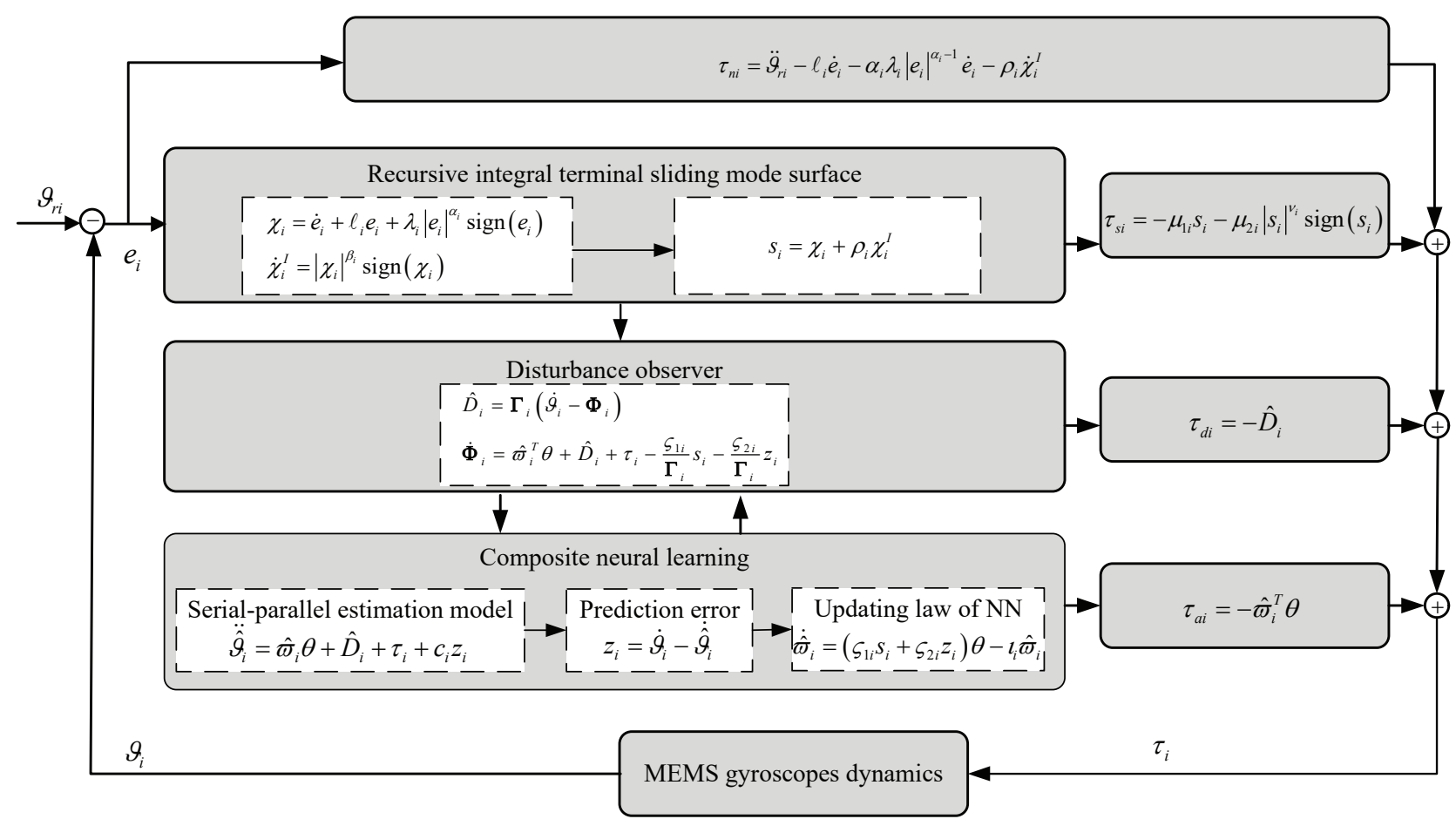

Fig. 2 Control scheme of the RTSMC for MEMS gyroscopes with composite learning and DOB.

where $\mu_{1 i}>0, \mu_{2 i}>0$ and $0<v_{i}<1$ are user-defined constants, and $\hat{D}_{i}$ is the estimation of the compound disturbance $D_{i}=d_{i}+\varepsilon_{i}$.

From the controller (11), $\dot{s}_{i}$ is calculated as

$\dot{s}_{i}=\tilde{\varpi}_{i}^{T} \theta+\tilde{D}_{i}-\mu_{1 i} s_{i}-\mu_{2 i}\left|s_{i}\right|^{v_{i}} \operatorname{sign}\left(s_{i}\right)$

where $\tilde{\varpi}_{i}=\bar{\varpi}_{i}-\hat{\varpi}_{i}, \tilde{D}_{i}=D_{i}-\hat{D}_{i}$.

The prediction error $z_{i}$ is constructed as

$z_{i}=\dot{\vartheta}_{i}-\dot{\hat{\vartheta}}_{i}$

where $\dot{\hat{\vartheta}}_{i}$ is the estimation of $\dot{\vartheta}_{i}$, and $\dot{\hat{\vartheta}}_{i}$ is given by the following SPEM:

$\ddot{\hat{\vartheta}}_{i}=\hat{\emptyset}_{i}^{T} \theta+\hat{D}_{i}+\tau_{i}+c_{i} z_{i}$

The derivative of $z_{i}$ is calculated as

$$
\begin{aligned}
\dot{z}_{i} & =\ddot{\vartheta}_{i}-\ddot{\hat{\vartheta}}_{i} \\
& =\widetilde{\varpi}_{i}^{T} \theta+D_{i}+\tau_{i}-\left(\hat{\varpi}_{i}^{T} \theta+\hat{D}_{i}+\tau_{i}+c_{i} z_{i}\right) \\
& =\tilde{\varpi}_{i}^{T} \theta+\tilde{D}_{i}-c_{i} z_{i}
\end{aligned}
$$

The updating law is proposed as

$\dot{\hat{\sigma}}_{i}=\left(\varsigma_{1 i} s_{i}+\varsigma_{2 i} z_{i}\right) \theta-\imath_{i} \hat{\bar{\omega}}_{i}$

The DOB is designed as

$$
\begin{aligned}
& \hat{D}_{i}=\Gamma_{i}\left(\dot{\vartheta}_{i}-\Phi_{i}\right) \\
& \dot{\Phi}_{i}=\hat{\omega}_{i}^{T} \theta+\hat{D}_{i}+\tau_{i}-\frac{\varsigma_{1 i}}{\Gamma_{i}} s_{i}-\frac{\varsigma_{2 i}}{\Gamma_{i}} z_{i}
\end{aligned}
$$

where $\Gamma_{i}>0$ is an user-defined constant, and $\Phi_{i}$ is the intermediate variable.
The derivative of $\tilde{D}_{i}$ is calculated as

$$
\begin{aligned}
\dot{\tilde{D}}_{i}= & \dot{D}_{i}-\dot{\hat{D}}_{i} \\
= & \dot{D}_{i}-\Gamma_{i}\left(\ddot{\vartheta}_{i}-\dot{\Phi}_{i}\right) \\
= & \dot{D}_{i}-\Gamma_{i}\left(\varpi_{i}^{T} \theta+D_{i}+\tau_{i}\right) \\
& +\Gamma_{i}\left(\hat{\varpi}_{i}^{T} \theta+\hat{D}_{i}+\tau_{i}-\frac{\varsigma_{1 i}}{\Gamma_{i}} s_{i}-\frac{\varsigma_{2 i}}{\Gamma_{i}} z_{i}\right) \\
= & \dot{D}_{i}-\Gamma_{i} \tilde{\varpi}_{i}^{T} \theta-\Gamma_{i} \tilde{D}_{i}-\varsigma_{1 i} s_{i}-\varsigma_{2 i} z_{i}
\end{aligned}
$$

Remark 3 In the controller (11), $\tau_{a i}$ is the adaptive control using composite neural learning to approximate the system nonlinearity, $\tau_{d i}$ is the DOB designed to deal with the external disturbances, $\tau_{n i}$ is the nominal feedback controller, $\tau_{s i}$ is the RTSMC signal to achieve finite time convergence.

Remark 4 Since the approximation error $\varepsilon_{i}$ and its derivative $\dot{\varepsilon}_{i}$ are bounded, the boundedness of the compound disturbance $D_{i}$ and its derivative $\dot{D}_{i}$ can be guaranteed under Assumption1.

Remark 5 It is observed from the sliding mode manifold (8) that $s_{i}(0)=0$ when $\chi_{i}^{I}(0)=-\chi_{i}(0) / \rho_{i}$, where $s_{i}(0)$, $\chi_{i}^{I}(0)$ and $\chi_{i}(0)$ are the initial values of $s_{i}, \chi_{i}^{I}$ and $\chi_{i}$, respectively. That implies faster convergence will be achieved when the initial value of $\chi_{i}^{I}$ is chosen as $\chi_{i}^{I}(0)=-\left[\dot{e}_{i}(0)+\right.$ $\left.\ell_{i} e_{i}(0)+\lambda_{i}\left|e_{i}(0)\right|^{\alpha_{i}} \operatorname{sign}\left(e_{i}(0)\right)\right] / \rho_{i}$, because the control system is forced to start from the sliding surface at the initial time. 


\section{Stability Analysis}

Lemma 1 For the dynamics (1), if there exists the Lyapunov function $V$ satisfying $\dot{V}+\gamma_{1} V+\gamma_{2} V^{\gamma_{m}} \leq 0$, the system stability can be guaranteed in the finite time $T_{0}[30]$, with $\gamma_{1}>0$, $\gamma_{2}>0,0<\gamma_{m}<1$, and

$T_{0}=\frac{1}{\gamma_{1}\left(1-\gamma_{m}\right)} \ln \left(\frac{\gamma_{1} V^{1-\gamma_{m}}(0)+\gamma_{2}}{\gamma_{2}}\right)$

where $V(0)$ is the initial value of $V$.

Theorem 1 Considering the dimensionless dynamics of MEM$S$ gyroscopes described by (1), if the controller (11), the composite learning (17) and the DOB (18) are designed, the signals in (21) are bounded, and the tracking error $e_{i}$ will converge to the region $\aleph_{i}$ in the finite time $T_{i}$.

Proof: The Lyapunov function candidate is selected as $V=\sum_{i=x, y} V_{i}$

where $V_{i}=V_{s_{i}}+V_{z_{i}}+V_{\tilde{\omega}_{i}}+V_{\tilde{D}_{i}}$, with

$V_{s_{i}}=\frac{\varsigma_{1 i}}{2} s_{i}^{2}, V_{z_{i}}=\frac{\varsigma_{2 i}}{2} z_{i}^{2}, V_{\tilde{\varpi}_{i}}=\frac{1}{2} \tilde{\varpi}_{i}^{T} \tilde{\varpi}_{i}, V_{\tilde{D}_{i}}=\frac{1}{2} \tilde{D}_{i}^{2}$

The derivative of $V_{s_{i}}$ is calculated as

$\dot{V}_{s_{i}}=\varsigma_{1 i} s_{i} \dot{s}_{i}$

$$
\begin{aligned}
& =\varsigma_{1 i} s_{i}\left[\tilde{\varpi}_{i}^{T} \theta+\tilde{D}_{i}-\mu_{1 i} s_{i}-\mu_{2 i}\left|s_{i}\right|^{v_{i}} \operatorname{sign}\left(s_{i}\right)\right] \\
& =\varsigma_{1 i} s_{i}\left(\tilde{\tilde{\omega}}_{i}^{T} \theta+\tilde{D}_{i}\right)-\varsigma_{1 i} \mu_{1 i} s_{i}^{2}-\varsigma_{1 i} \mu_{2 i}\left|s_{i}\right|^{v_{i}+1}
\end{aligned}
$$

The derivative of $V_{z_{i}}$ is calculated as

$$
\begin{aligned}
\dot{V}_{z_{i}} & =\varsigma_{2 i} z_{i} \dot{z}_{i} \\
& =\varsigma_{2 i} z_{i}\left(\tilde{\varpi}_{i}^{T} \theta+\tilde{D}_{i}-c_{i} z_{i}\right) \\
& =\varsigma_{2 i} z_{i}\left(\tilde{\varpi}_{i}^{T} \theta+\tilde{D}_{i}\right)-\varsigma_{2 i} c_{i} z_{i}^{2}
\end{aligned}
$$

The derivative of $V_{\tilde{\omega}_{i}}$ is calculated as

$$
\begin{aligned}
\dot{V}_{\tilde{\tilde{\omega}}_{i}} & =\tilde{\varpi}_{i}^{T} \dot{\tilde{\varpi}}_{i} \\
& =-\tilde{\varpi}_{i}^{T} \dot{\hat{\varpi}}_{i} \\
& =-\tilde{\varpi}_{i}^{T}\left[\left(\varsigma_{1 i} s_{i}+\varsigma_{2 i} z_{i}\right) \theta-\imath_{i} \hat{\varpi}_{i}\right]
\end{aligned}
$$

The derivative of $V_{\tilde{D}_{i}}$ is calculated as

$$
\begin{aligned}
\dot{V}_{\tilde{D}_{i}} & =\tilde{D}_{i} \dot{\tilde{D}}_{i} \\
& =\tilde{D}_{i}\left(\dot{D}_{i}-\Gamma_{i} \tilde{\varpi}_{i}^{T} \theta-\Gamma_{i} \tilde{D}_{i}-\varsigma_{1 i} s_{i}-\varsigma_{2 i} z_{i}\right)
\end{aligned}
$$

Then we obtain

$$
\begin{aligned}
\dot{V}_{i}= & \dot{V}_{s_{i}}+\dot{V}_{z_{i}}+\dot{V}_{\tilde{\varpi}_{i}}+\dot{V}_{\tilde{D}_{i}} \\
= & \varsigma_{1 i} s_{i}\left(\tilde{\varpi}_{i}^{T} \theta+\tilde{D}_{i}\right)-\varsigma_{1 i} \mu_{1 i} s_{i}^{2}-\varsigma_{1 i} \mu_{2 i}\left|s_{i}\right|^{v_{i}+1} \\
& +\varsigma_{2 i} z_{i}\left(\tilde{\varpi}_{i}^{T} \theta+\tilde{D}_{i}\right)-\varsigma_{2 i} c_{i} z_{i}^{2} \\
& -\tilde{\varpi}_{i}^{T}\left[\left(\varsigma_{1 i} s_{i}+\varsigma_{2 i} z_{i}\right) \theta-\iota_{i} \hat{\varpi}_{i}\right] \\
& +\tilde{D}_{i}\left(\dot{D}_{i}-\Gamma_{i} \tilde{\varpi}_{i}^{T} \theta-\Gamma_{i} \tilde{D}_{i}-\varsigma_{1 i} s_{i}-\varsigma_{2 i} z_{i}\right) \\
= & -\varsigma_{1 i} \mu_{1 i} s_{i}^{2}-\varsigma_{1 i} \mu_{2 i}\left|s_{i}\right|^{v_{i}+1}-\varsigma_{2 i} c_{i} z_{i}^{2} \\
& +\iota_{i} \tilde{\varpi}_{i}^{T} \hat{\varpi}_{i}+\tilde{D}_{i}\left(\dot{D}_{i}-\Gamma_{i} \tilde{\varpi}_{i}^{T} \theta-\Gamma_{i} \tilde{D}_{i}\right)
\end{aligned}
$$

Since the following facts exist

$$
\begin{aligned}
\tilde{\varpi}_{i}^{T} \hat{\varpi}_{i} & \leq \frac{1}{2}\left\|\varpi_{i}\right\|^{2}-\frac{1}{2}\left\|\tilde{\varpi}_{i}\right\|^{2} \\
\tilde{D}_{i} \dot{D}_{i} & \leq \frac{1}{2} \tilde{D}_{i}^{2}+\frac{1}{2} \dot{D}_{i}^{2} \\
-\tilde{D}_{i} \tilde{\varpi}_{i}^{T} \theta & \leq \frac{1}{2} c_{m} c_{\theta}^{2} \tilde{D}_{i}^{2}+\frac{1}{2 c_{m}}\left\|\tilde{\varpi}_{i}\right\|^{2}
\end{aligned}
$$

where $\|\theta\| \leq c_{\theta}$, and $c_{m}>0$ is a constant.

$\dot{V}_{i}$ is rewritten as

$$
\begin{aligned}
\dot{V}_{i} \leq & -\varsigma_{1 i} \mu_{1 i} s_{i}^{2}-\varsigma_{1 i} \mu_{2 i}\left|s_{i}\right|^{v_{i}+1}-\varsigma_{2 i} c_{i} z_{i}^{2}+\frac{l_{i}}{2}\left\|\varpi_{i}\right\|^{2} \\
& -\frac{l_{i}}{2}\left\|\tilde{\varpi}_{i}\right\|^{2}+\frac{1}{2} \tilde{D}_{i}^{2}+\frac{1}{2} \dot{D}_{i}^{2}+\frac{1}{2} \Gamma_{i} c_{m} c_{\theta}^{2} \tilde{D}_{i}^{2} \\
& +\frac{1}{2 c_{m}} \Gamma_{i}\left\|\tilde{\varpi}_{i}\right\|^{2}-\Gamma_{i} \tilde{D}_{i}^{2} \\
\leq & -\varsigma_{1 i} \mu_{1 i} s_{i}^{2}-\varsigma_{1 i} \mu_{2 i}\left|s_{i}\right|^{v_{i}+1}-\varsigma_{2 i} c_{i} z_{i}^{2} \\
& -\frac{1}{2}\left(\imath_{i}-\frac{\Gamma_{i}}{c_{m}}\right)\left\|\tilde{\omega}_{i}\right\|^{2}+\frac{1}{2}\left(\Gamma_{i} c_{m} c_{\theta}^{2}-2 \Gamma_{i}+1\right) \tilde{D}_{i}^{2} \\
& +\frac{l_{i}}{2}\left\|{\varpi_{i}}_{i}\right\|^{2}+\frac{1}{2} \dot{D}_{i}^{2} \\
= & -\varsigma_{1 i} \mu_{1 i} s_{i}^{2}-\varsigma_{1 i} \mu_{2 i}\left|s_{i}\right|^{v_{i}+1}-\varsigma_{2 i} c_{i} z_{i}^{2} \\
& -\frac{1}{2} C_{\tilde{\omega}_{i}}\left\|\tilde{\varpi}_{i}\right\|^{2}-\frac{1}{2} C_{\tilde{D}_{i}} \tilde{D}_{i}^{2}+C_{V}
\end{aligned}
$$

where $C_{\tilde{\omega}_{i}}=\imath_{i}-\Gamma_{i} / c_{m}, C_{\tilde{D}_{i}}=2 \Gamma_{i}-\Gamma_{i} c_{m} c_{\theta}^{2}-1, C_{V}=\imath_{i}\left\|\varpi_{i}\right\|^{2} / 2+$ $\dot{D}_{i}^{2} / 2$. Parameters $\boldsymbol{\iota}_{i}, \Gamma_{i}, c_{m}$ are selected to force $C_{\tilde{\omega}_{i}} \geq 0$ and $C_{\tilde{D}_{i}} \geq 0$.

Furthermore, it is concluded that

$\dot{V}_{i} \leq-\varsigma_{2 i} c_{i} z_{i}^{2}-\frac{1}{2} C_{\tilde{\omega}_{i}}\left\|\tilde{\varpi}_{i}\right\|^{2}-\frac{1}{2} C_{\tilde{D}_{i}} \tilde{D}_{i}^{2}+C_{V}$

Hence, $z_{i}, \tilde{\varpi}_{i}$ and $\tilde{D}_{i}$ are bounded with $\left|z_{i}\right| \leq \sqrt{C_{V} / \varsigma_{2 i} c_{i}}$, $\left\|\tilde{\omega}_{i}\right\| \leq \sqrt{2 C_{V} / C_{\tilde{\omega}_{i}}}$ and $\left|\tilde{D}_{i}\right| \leq \sqrt{2 C_{V} / C_{\tilde{D}_{i}}}$.

Define $C_{b}=\tilde{\varpi}_{i}^{T} \theta+\tilde{D}_{i}$. Since $\tilde{\varpi}_{i}$ and $\tilde{D}_{i}$ are bounded, there exists an unknown constant $B_{m}>0$ such that $\left|C_{b}\right| \leq$ $B_{m}$. From (23), it is concluded that

$$
\begin{aligned}
\dot{V}_{s_{i}}= & \varsigma_{1 i} s_{i} C_{b}-\varsigma_{1 i} \mu_{1 i} s_{i}^{2}-\varsigma_{1 i} \mu_{2 i}\left|s_{i}\right|^{v_{i}+1} \\
\leq & -\varsigma_{1 i}\left(\mu_{1 i}-\frac{B_{m}}{2\left|s_{i}\right|}\right) s_{i}^{2} \\
& -\varsigma_{1 i}\left(\mu_{2 i}-\frac{B_{m}}{2\left|s_{i}\right| v_{i}}\right)\left|s_{i}\right|^{v_{i}+1}
\end{aligned}
$$

If $s_{i} \neq 0$, it is obtained that

$$
\dot{V}_{s_{i}} \leq-\bar{\mu}_{1 i} V_{s_{i}}-\bar{\mu}_{2 i} V_{s_{i}}^{\frac{v_{i}+1}{2}}
$$

with

$$
\begin{aligned}
& \bar{\mu}_{1 i}=2\left(\mu_{1 i}-\frac{B_{m}}{2\left|s_{i}\right|}\right) \\
& \bar{\mu}_{2 i}=2\left(\frac{2}{\varsigma_{1 i}}\right)^{\frac{v_{i}-1}{2}}\left(\mu_{2 i}-\frac{B_{m}}{2\left|s_{i}\right|^{v_{i}}}\right)
\end{aligned}
$$


From Lemma 1, it is known that $\left|s_{i}\right|$ will converge to the region $\Lambda_{i}=\min \left\{\Lambda_{1 i}, \Lambda_{2 i}\right\}$ in finite time $T_{s i}$, with

$$
\begin{aligned}
& \Lambda_{1 i}=\left\{\left|s_{i}\right| \leq \frac{B_{m}}{2 \mu_{1 i}}\right\} \\
& \Lambda_{2 i}=\left\{\left|s_{i}\right| \leq\left(\frac{B_{m}}{2 \mu_{2 i}}\right)^{\frac{1}{v_{i}}}\right\} \\
& T_{s i} \leq \frac{2}{\bar{\mu}_{1 i}\left(1-v_{i}\right)} \ln \left(\frac{\bar{\mu}_{1 i} V_{s i}^{\frac{1-v_{i}}{2}}(0)+\bar{\mu}_{2 i}}{\bar{\mu}_{2 i}}\right)
\end{aligned}
$$

where $V_{s i}(0)$ is the initial value of $V_{s i}$.

When $s_{i}=0$, there exists $\chi_{i}=-\rho_{i} \chi_{i}^{I}$, such that $\chi_{i}$ is with the same convergence time as $\chi_{i}^{I}$. Define $V_{\chi_{i}^{I}}=\left(\chi_{i}^{I}\right)^{2} / 2$. It is calculated that

$$
\begin{aligned}
\dot{V}_{\chi_{i}^{I}} & =\chi_{i}^{I} \dot{\chi}_{i}^{I} \\
& =\chi_{i}^{I}\left|\chi_{i}\right|^{\beta_{i}} \operatorname{sign}\left(\chi_{i}\right) \\
& =\chi_{i}^{I}\left|s_{i}-\rho_{i} \chi_{i}^{I}\right|^{\beta_{i}} \operatorname{sign}\left(s_{i}-\rho_{i} \chi_{i}^{I}\right)
\end{aligned}
$$

When $\left|\rho_{i} \chi_{i}^{I}\right| \geq\left|s_{i}\right|+C_{s i}$, where $C_{s i}>0$ is an unknown constant, $\dot{V}_{\chi_{i}^{I}}$ can be rewritten as

$$
\begin{aligned}
\dot{V}_{\chi_{i}^{I}} & =-\chi_{i}^{I}\left|s_{i}-\rho_{i} \chi_{i}^{I}\right|^{\beta_{i}} \operatorname{sign}\left(\chi_{i}^{I}\right) \\
& \leq-\left|\chi_{i}^{I}\right|\left(\left|\rho_{i} \chi_{i}^{I}\right|^{\beta_{i}}-\left|s_{i}\right|^{\beta_{i}}\right) \\
& \leq-C_{s i}^{\beta_{i}}\left|\chi_{i}^{I}\right| \\
& =-\sqrt{2} C_{s i}^{\beta_{i}} V_{\chi_{i}^{I}}^{\frac{1}{2}}
\end{aligned}
$$

Referring to $[31,12]$, it is concluded that $\chi_{i}^{I}$ will converge to the region $\Lambda_{\chi_{i}^{I}}$ in finite time $T_{\chi_{i}}$ when $\left|s_{i}\right|$ is in the region $\Lambda_{i}$, where

$$
\begin{aligned}
& \Lambda_{\chi_{i}^{I}}=\left\{\left|\chi_{i}^{I}\right| \leq \frac{\Lambda_{i}+C_{s i}}{\rho_{i}}\right\} \\
& T_{\chi_{i}} \leq \frac{V_{\chi_{i}^{I}}^{1-\frac{1}{2}}(0)}{\sqrt{2} C_{s i}^{\beta_{i}}\left(1-\frac{1}{2}\right)}=\frac{\sqrt{2} V_{\chi_{i}^{I}}^{\frac{1}{2}}(0)}{C_{s i}^{\beta_{i}}}
\end{aligned}
$$

Hence, $\left|\chi_{i}\right|$ will converge to the region $\Lambda_{\chi_{i}}=\Lambda_{i}+C_{s i}$ in finite time $T_{\chi_{i}}$. Similar to $[31,12]$, it is easy to verify that the above finite time convergence still can be guaranteed when $\left|\rho_{i} \chi_{i}^{I}\right|<\left|s_{i}\right|+C_{s i}$.

Defining $V_{e_{i}}=e_{i}^{2} / 2$, we have

$$
\begin{aligned}
\dot{V}_{e_{i}} & =e_{i} \dot{e}_{i} \\
& =e_{i}\left[\chi_{i}-\ell_{i} e_{i}-\lambda_{i}\left|e_{i}\right|^{\alpha_{i}} \operatorname{sign}\left(e_{i}\right)\right] \\
& =e_{i} \chi_{i}-\ell_{i} e_{i}^{2}-\lambda_{i}\left|e_{i}\right|^{\alpha_{i}+1} \\
& \leq-\left(\ell_{i}-\frac{\Lambda_{\chi_{i}}}{2\left|e_{i}\right|}\right) e_{i}^{2}-\left(\lambda_{i}-\frac{\Lambda_{\chi_{i}}}{2\left|e_{i}\right|^{\alpha_{i}}}\right)\left|e_{i}\right|^{\alpha_{i}+1} \\
& \text { If } e_{i} \neq 0, \text { it is obtained that }
\end{aligned}
$$$$
\dot{V}_{e_{i}} \leq-\mathrm{E}_{1 i} V_{e_{i}}-\mathrm{E}_{2 i} V_{e_{i}}^{\frac{\alpha_{i}+1}{2}}
$$

with

$$
\begin{aligned}
& \mathrm{E}_{1 i}=2\left(\ell_{i}-\frac{\Lambda_{\chi_{i}}}{2\left|e_{i}\right|}\right) \\
& \mathrm{E}_{2 i}=2^{\frac{\alpha_{i}+1}{2}}\left(\lambda_{i}-\frac{\Lambda_{\chi_{i}}}{2\left|e_{i}\right| \alpha_{i}}\right)
\end{aligned}
$$

From Lemma 1, it is known that $\left|e_{i}\right|$ will converge to the region $\boldsymbol{\aleph}_{i}=\min \left\{\boldsymbol{\aleph}_{1 i}, \boldsymbol{\aleph}_{2 i}\right\}$ in finite time $T_{e i}$ when $\left|\chi_{i}\right|$ is in the region $\Lambda_{\chi_{i}}$, with

$$
\begin{aligned}
\boldsymbol{\aleph}_{1 i} & =\left\{\left|e_{i}\right| \leq \frac{\Lambda_{\chi_{i}}}{2 \ell_{i}}\right\} \\
\boldsymbol{\aleph}_{2 i} & =\left\{\left|e_{i}\right| \leq\left(\frac{\Lambda_{\chi_{i}}}{2 \lambda_{i}}\right)^{\frac{1}{\alpha_{i}}}\right\} \\
T_{e i} & \leq \frac{2}{\mathrm{E}_{1 i}\left(1-\alpha_{i}\right)} \ln \left(\frac{\mathrm{E}_{1 i} V_{e i}^{\frac{1-\alpha_{i}}{2}}(0)+\mathrm{E}_{2 i}}{\mathrm{E}_{2 i}}\right)
\end{aligned}
$$

where $V_{e i}(0)$ is the initial value of $V_{e i}$.

Thus, $e_{i}$ converges from any initial condition to the region $\aleph_{i}$ in the finite time $T_{i}=T_{s i}+T_{\chi_{i}}+T_{e i}$.

\section{Simulation}

Note that the proposed controller (11) is denoted as "CLRTSMC". The highlights of "CL-RTSMC" are the RTSMC (8) and the composite neural learning (17). To verify faster convergence and higher tracking accuracy of "CL-RTSMC", it is compared with "CL-TSMC" (the controller not using the RTSMC) and "NN-TSMC" (the controller not using the RTSMC and the composite neural learning) in simulations, while "CL-TSMC" and "NN-TSMC" are presented as follows:

(a) "CL-TSMC": The sliding mode manifold is designed as

$s_{i}=\dot{e}_{i}+\ell_{i} e_{i}+\lambda_{i}\left|e_{i}\right|^{\alpha_{i}} \operatorname{sign}\left(e_{i}\right)$

The controller is designed as

$\tau_{i}=\tau_{a i}+\tau_{d i}+\tau_{n i}+\tau_{s i}$

with

$$
\begin{aligned}
\tau_{a i} & =-\hat{\varpi}_{i}^{T} \theta \\
\tau_{d i} & =-\hat{D}_{i} \\
\tau_{n i} & =\ddot{\vartheta}_{r i}-\ell_{i} \dot{e}_{i}-\alpha_{i} \lambda_{i}\left|e_{i}\right|^{\alpha_{i}-1} \dot{e}_{i} \\
\tau_{s i} & =-\mu_{1 i} s_{i}-\mu_{2 i}\left|s_{i}\right|^{v_{i}} \operatorname{sign}\left(s_{i}\right)
\end{aligned}
$$

where the DOB is designed as (18) and the updating law of $\mathrm{NN}$ weight is designed as

$\dot{\hat{\sigma}}_{i}=\left(\varsigma_{1 i} s_{i}+\varsigma_{2 i} z_{i}\right) \theta-\imath_{i} \hat{\bar{\omega}}_{i}$

with $z_{i}=\dot{\vartheta}_{i}-\dot{\hat{\vartheta}}_{i}$ constructed by the dynamics (1) and the SPEM (15). 
(b) "NN-TSMC": The sliding mode manifold is designed as (42). The controller is designed as (43). The DOB is designed as

$$
\begin{aligned}
& \hat{D}_{i}=\Gamma_{i}\left(\dot{\vartheta}_{i}-\Phi_{i}\right) \\
& \dot{\Phi}_{i}=\hat{\varpi}_{i}^{T} \theta+\hat{D}_{i}+\tau_{i}-\frac{\varsigma_{1 i}}{\Gamma_{i}} s_{i}
\end{aligned}
$$

and the updating law of $\mathrm{NN}$ weight is designed as

$\dot{\hat{\varpi}}_{i}=\varsigma_{1 i} s_{i} \theta-\imath_{i} \hat{\varpi}_{i}$

The dimensionless dynamics parameters [12] are given as $\varsigma_{x x}^{0}=0.075, \varsigma_{x y}^{0}=0.0075, \varsigma_{y x}^{0}=0.0075, \varsigma_{y y}^{0}=0.0075$, $\psi_{x x}^{0}=14207, \psi_{x y}^{0}=877, \psi_{y x}^{0}=877, \psi_{y y}^{0}=12564, \psi_{x 3}^{0}=$ $6.25 \times 10^{-4}, \psi_{y 3}^{0}=6.25 \times 10^{-4}$ and $\Omega=0.005$. The parameter uncertainties $\Delta \varsigma_{x x}, \Delta \varsigma_{x y}, \Delta \varsigma_{y x}$ and $\Delta \varsigma_{y y}$ are set as $5 \%$ of the nominal parts $\varsigma_{x x}^{0}, \varsigma_{x y}^{0}, \varsigma_{y x}^{0}$ and $\varsigma_{y y}^{0}$ respectively. The parameter uncertainties $\Delta \psi_{x x}, \Delta \psi_{x y}, \Delta \psi_{y x}, \Delta \psi_{y y}, \Delta \psi_{x 3}$ and $\Delta \psi_{y 3}$ are set as $0.25 \%$ of the nominal parts $\psi_{x x}^{0}, \psi_{x y}^{0}, \psi_{y x}^{0}$, $\psi_{y y}^{0}, \psi_{x 3}^{0}$ and $\psi_{y 3}^{0}$, respectively. The external disturbances are set as $d_{x}=0.7448+0.98 \sin (10.5 t)$ and $d_{y}=0.7448+$ $0.98 \sin (11 t)$. The reference signals are selected as $\vartheta_{r x}=$ $6.2 \sin (3.7685 t+\pi / 3)$ and $\vartheta_{r y}=5 \sin (3.5447 t-\pi / 6)$.

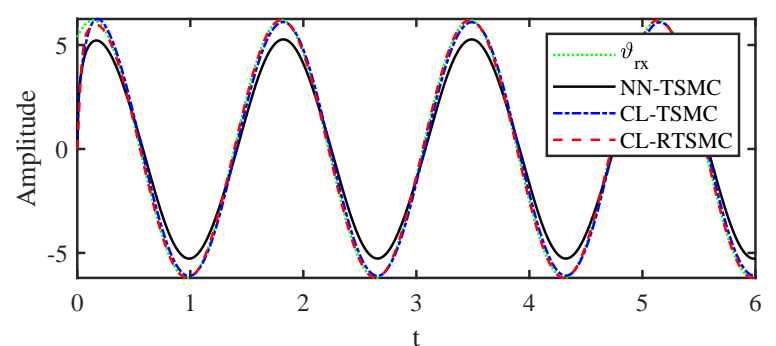

(a)

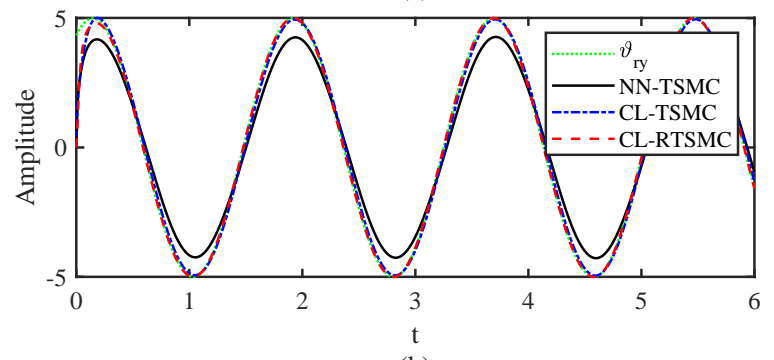

(b)

Fig. 3 System tracking: (a) $\vartheta_{r x}$ and $\vartheta_{x}$. (b) $\vartheta_{r y}$ and $\vartheta_{y}$.

For "NN-TSMC", the control parameters are set as $\mu_{1 i}=$ 200, $\mu_{2 i}=150, \alpha_{i}=2.4, v_{i}=0.9, \ell_{i}=10, \lambda_{i}=10, \Gamma_{i}=20$, $\iota_{i}=4$ and $\varsigma_{1 i}=810$. For "CL-TSMC", the same parameters are set as "NN-TSMC", while $\varsigma_{2 i}=54$ and $c_{i}=2$. For "CL-RTSMC", the same parameters are set as "NN-TSMC" and "CL-TSMC", while $\beta_{i}=0.6$ and $\rho_{i}=1000$. To further obtain faster convergence, the initial value of $\chi_{i}^{I}$ is chosen as $\chi_{i}^{I}(0)=-\left[\dot{e}_{i}(0)+\ell_{i} e_{i}(0)+\lambda_{i}\left|e_{i}(0)\right|^{\alpha_{i}} \operatorname{sign}\left(e_{i}(0)\right)\right] / \rho_{i}$.

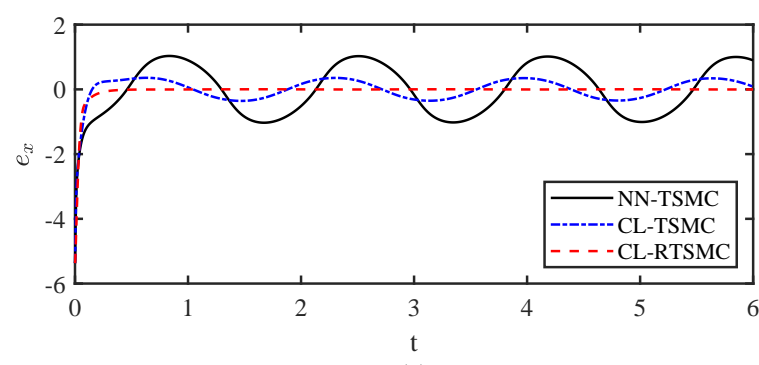

(a)

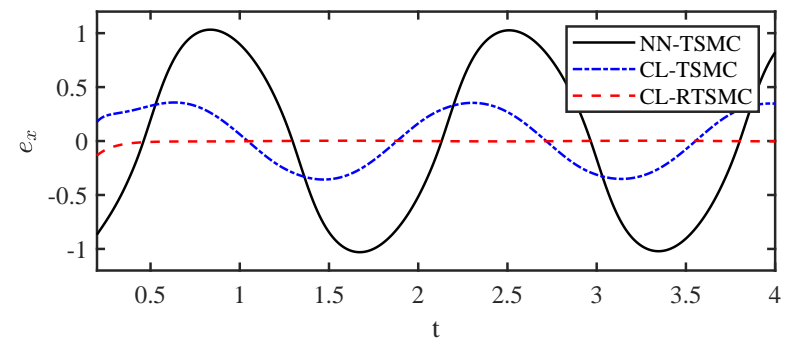

(b)

Fig. 4 Tracking errors of drive axis: (a) $e_{x}$. (b) $e_{x} \in[0.2,4]$.

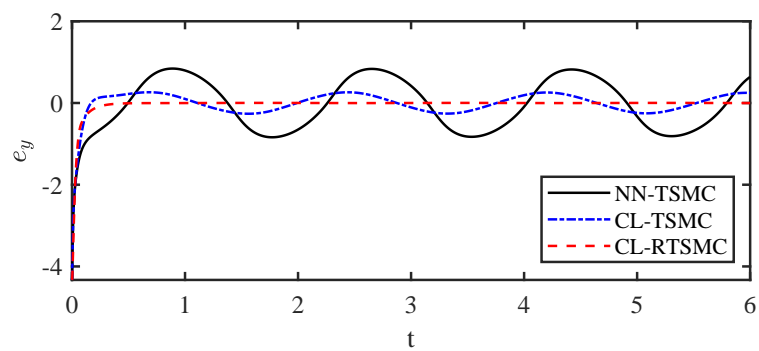

(a)

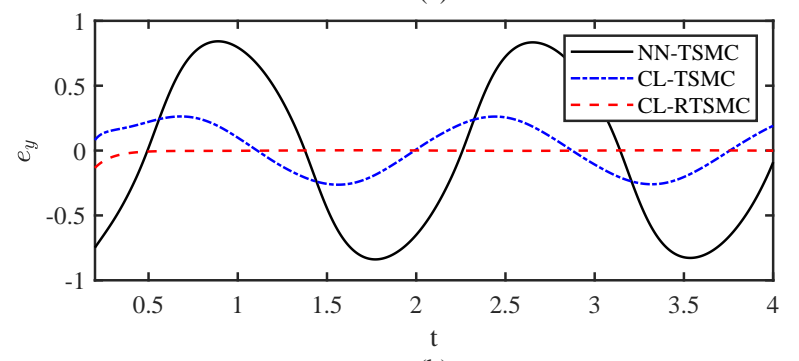

(b)

Fig. 5 Tracking errors of sensitive axis: (a) $e_{y}$. (b) $e_{y} \in[0.2,4]$.

The simulation results are demonstrated in Fig.3 Fig.10 and Table 1. It is observed from Fig.3 that system state $\vartheta_{i}$ can effectively follow the reference signal $\vartheta_{r i}$ under "NNTSMC", "CL-TSMC" and "CL-RTSMC", faster convergence and higher tracking accuracy are obtained under "CL-RTSMC" in Fig.4 Fig.5. To clearly analyze the tracking performance of controllers, the transient time, the average of steady-state errors and the root mean squard error (RMSE) of steadystate errors are presented in Table 1.

Through comparing the tracking performance index of "CL-TSMC" and "NN-TSMC', it is observed that, under 
Table 1 Tracking performance index of control schemes.

\begin{tabular}{c|c|c|c|c}
\hline $\begin{array}{c}\text { Tracking } \\
\text { error }\end{array}$ & Method & $\begin{array}{c}\text { Transient } \\
\text { time }\end{array}$ & $\begin{array}{c}\text { Average of } \\
\text { steady-state errors }\end{array}$ & $\begin{array}{c}\text { RMSE of } \\
\text { steady-state errors }\end{array}$ \\
\hline \multirow{3}{*}{$e_{x}$} & NN-TSMC & 0.5 & 0.0740 & 0.7604 \\
& CL-TSMC & 0.5 & 0.0246 & 0.2558 \\
& CL-RTSMC & 0.42 & $5.1836 \times 10^{-4}$ & 0.0028 \\
\hline \multirow{3}{*}{$e_{y}$} & NN-TSMC & 0.5 & 0.0144 & 0.6019 \\
& CL-TSMC & 0.5 & 0.0092 & 0.1838 \\
& CL-RTSMC & 0.4 & $3.9260 \times 10^{-4}$ & 0.0024 \\
\hline
\end{tabular}
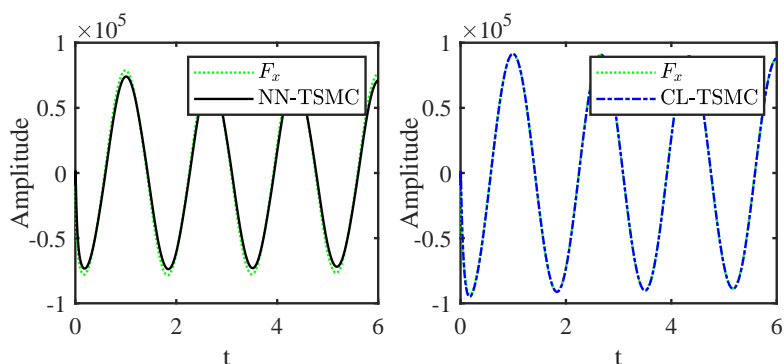

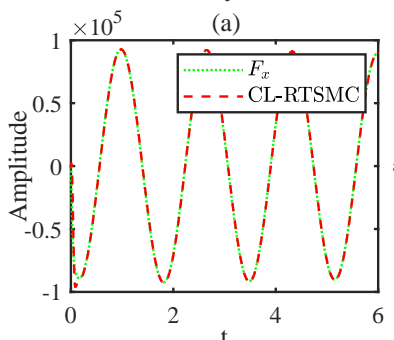

(c)

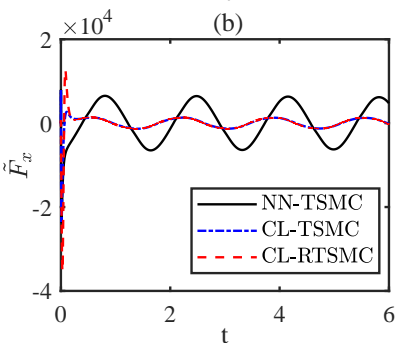

(d)
Fig. 6 Estimation of lumped uncertainty $F_{x}$ : (a) $F_{x}$ and $\hat{F}_{x}$ under "NNTSMC". (b) $F_{x}$ and $\hat{F}_{x}$ under "CL-TSMC". (c) $F_{x}$ and $\hat{F}_{x}$ under "CLRTSMC”. (d) Estimation error $\tilde{F}_{x}$.

"CL-TSMC', the average of steady-state errors in drive axis and sensitive axis are shortened by $66.76 \%$ and $36.11 \%$ respectively, while the RMSE of steady-state errors are shortened by $66.36 \%$ and $69.46 \%$ respectively. Higher tracking accuracy is obtained under "CL-TSMC" because the prediction error $z_{i}$ constructed from the SPEM is introduced to the updating law (17), such that the approximation error $\varepsilon_{i}$ of $\mathrm{NN}$ is indirectly considered. The estimation of the lumped uncertainty $F_{i}=f_{i}+d_{i}$ shown in Fig.6 Fig.7 further confirms the above conclusion, where smaller estimation error $\tilde{F}_{i}=\tilde{\varpi}_{i}^{T} \theta+\tilde{D}_{i}$ is obtained under "CL-TSMC"'.

Through comparing the tracking performance index of "CL-RTSMC" and "CL-TSMC', it is observed that, under "CL-RTSMC', the transient time is shortened by $16 \%$, the average of steady-state errors in drive axis and sensitive axis are shortened by $99.30 \%$ and $97.27 \%$ respectively, and the RMSE of steady-state errors are shortened by $99.63 \%$ and 99.60\% respectively. Higher tracking accuracy and faster convergence are achieved under RTSMC.

The norm of the RBF NN weight vector $\hat{\varpi}_{i}$ is shown in Fig.8, which is bounded in the steady state. From the s-
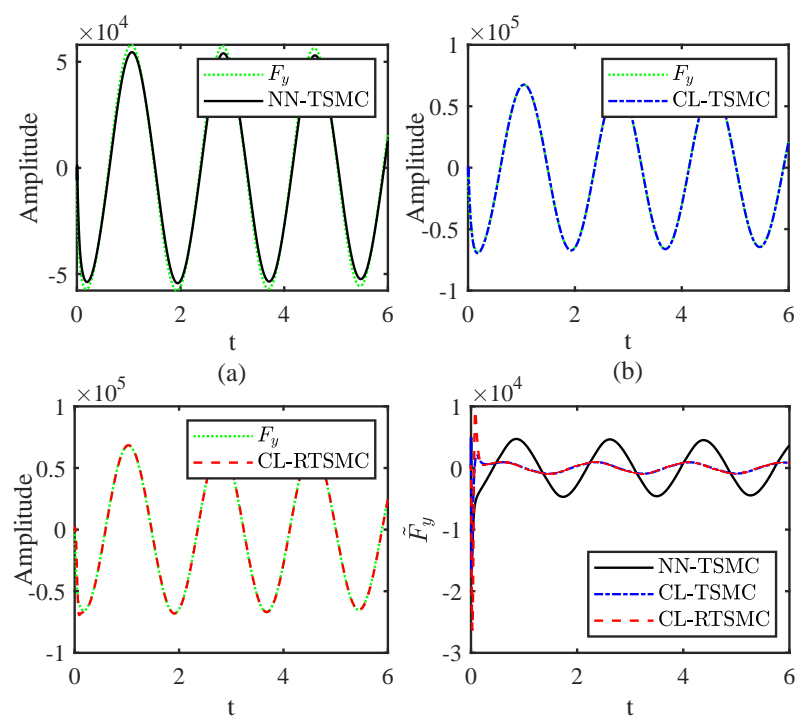

(c)

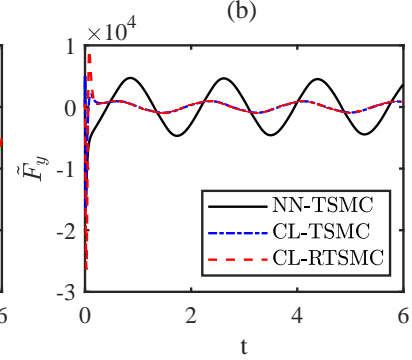

(d)
Fig. 7 Estimation of lumped uncertainty $F_{y}$ : (a) $F_{y}$ and $\hat{F}_{y}$ under "NNTSMC". (b) $F_{y}$ and $\hat{F}_{y}$ under "CL-TSMC". (c) $F_{y}$ and $\hat{F}_{y}$ under " CLRTSMC". (d) Estimation error $\tilde{F}_{y}$.

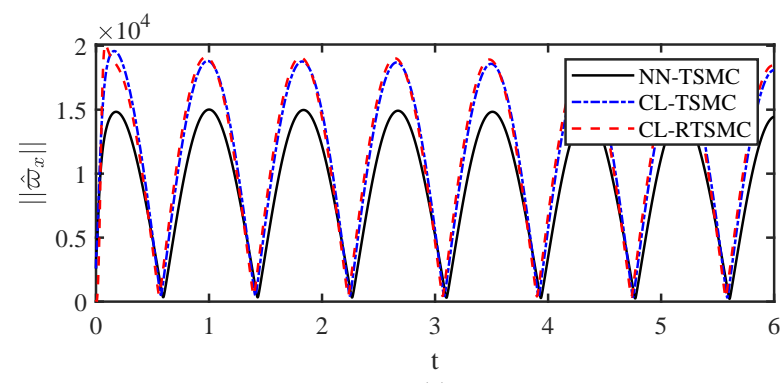

(a)

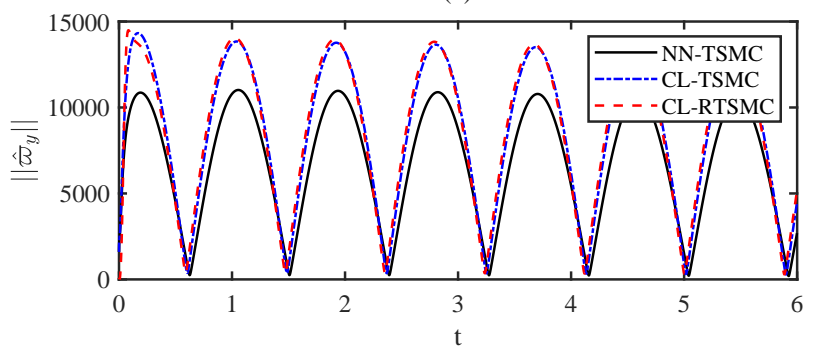

(b)

Fig. 8 Weight vector norm $\left\|\hat{\varpi}_{i}\right\|:$ (a) $\left\|\hat{\varpi}_{x}\right\|$. (b) $\left\|\hat{\varpi}_{y}\right\|$.

liding mode manifold $s_{i}$ presented in Fig.9, it is observed that the system states under "CL-RTSMC" are closer to the sliding mode. The control input $\tau_{i}$ under two methods are depicted in Fig.10, where $\tau_{i}$ has reached 100000 in some cases. It is noted that $\tau_{i}$ is the dimensionless control amoun$\mathrm{t}$ rather than the control force directly implemented on the MEMS gyroscopes. The dimensionless control amount $\tau_{i}$ is transformed from the control force $\tau_{i}^{*}$ to avoid the effec$\mathrm{t}$ of the dimension on gyroscopic motion characteristics by using the reference frequency $\omega_{0}=1 \mathrm{kHz}$, reference length 

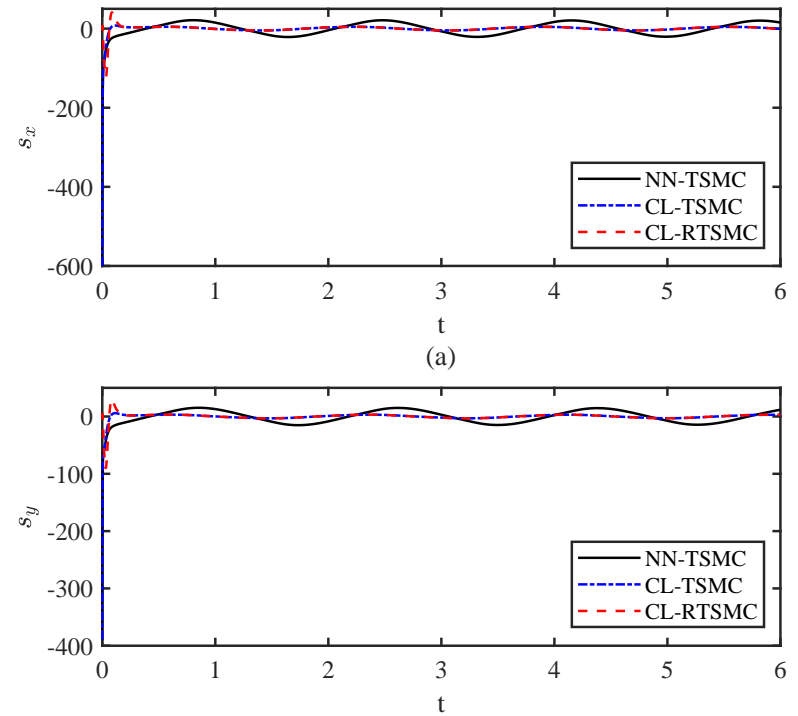

(b)

Fig. 9 Sliding mode manifold $s_{i}$ : (a) $s_{x}$. (b) $s_{y}$.
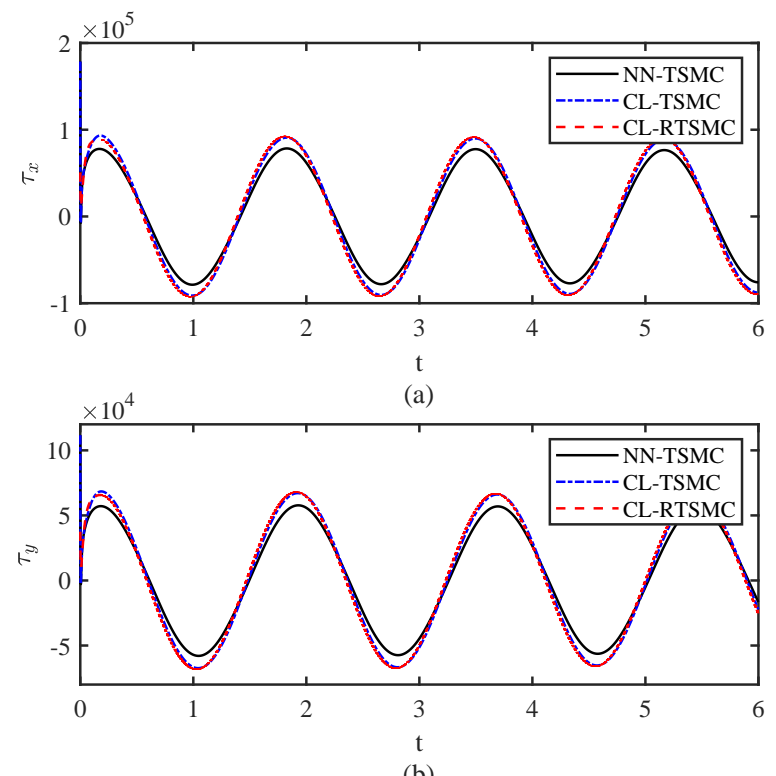

(b)

Fig. 10 Control input $\tau_{i}:$ (a) $\tau_{x}$. (b) $\tau_{y}$.

$\vartheta_{0}=10^{-5} \mathrm{~m}$ and the mass $m=5.7 \times 10^{-9} \mathrm{~kg}$, where the deducing process is presented in [25]. Thus, it is referred that $\tau_{i}^{*}=m \omega_{0}^{2} \vartheta_{0} \tau_{i}=5.7 \times 10^{-8} \tau_{i}$. When $\tau_{i}$ reaches 100000 , the control forces are calculated as $\tau_{i}^{*}=5.7 \times 10^{-3} \mathrm{~N}$. For MEMS gyroscopes, the actual control force $\tau_{i}^{*}$ meets the requirement. Hence, $\tau_{i}$ reaching 100000 in some cases is reasonable. Thus the simulation results verified that the expected higher tracking accuracy of the proposed RTSMC using the composite neural learning and the DOB can be obtained.

\section{Conclusions}

In this paper, the RTSMC has been designed to control the dynamics of MEMS gyroscopes with faster convergence and higher tracking accuracy in the presence of system uncertainties and external disturbances. In the proposed method, system states have been forced to start from the sliding mode surface at the initial time. The composite neural learning based on SPEM has been proposed to improve the approximation accuracy of system nonlinearity, such that higher tracking accuracy can be obtained. The DOB has been employed to handle the external disturbances. Simulation results have been demonstrated to verify that the proposed control can nicely achieve faster convergence and higher accuracy, compared with the schemes of "NN-TSMC" and "CL-TSMC".

Acknowledgements This work was supported by the National Natural Science Foundation of China under Grant 61933010.

\section{Data Availability Statement}

My manuscript has no associated data.

\section{Declarations}

Data Availability Statement The authors declare that they have no conflict of interest.

\section{References}

1. Park, S., Horowitz, R.: Adaptive control for the conventional mode of operation of MEMS gyroscopes. Journal of Microelectromechanical Systems 12(1), 101-108 (2003)

2. Leland, R.: Adaptive control of a MEMS gyroscope using lyapunov methods. IEEE Transactions on Control Systems Technology 14(2), 278-283 (2006)

3. Yue, F., Li, X.: Adaptive sliding mode control based on friction compensation for opto-electronic tracking system using neural network approximations. Nonlinear Dynamics 96(4), 2601-2612 (2019)

4. Wang, Y., Li, S., Zhang, B.: General regression neural networkbased data-driven model-free predictive functional control for a class of discrete-time nonlinear systems. Nonlinear Dynamics 107(1), 953-966 (2022)

5. Chu, Y., Fang, Y., Fei, J.: Adaptive neural dynamic global PID sliding mode control for MEMS gyroscope. International Journal of Machine Learning and Cybernetics 8(5), 1707-1718 (2017)

6. Shao, X., Shi, Y.: Neural-network-based constrained outputfeedback control for MEMS gyroscopes considering scarce transmission bandwidth. IEEE Transactions on Cybernetics DOI 10.1109/TCYB.2021.3070137

7. Guo, Y., Xu, B., Zhang, R.: Terminal sliding mode control of MEMS gyroscopes with finite-time learning. IEEE Transaction$\mathrm{s}$ on Neural Networks and Learning Systems 32(10), 4490-4498 (2021) 
8. Fei, J., Wang, Z.: Multi-loop recurrent neural network fractionalorder terminal sliding mode control of MEMS gyroscope. IEEE Access 8, 167965-167974 (2020)

9. Wang, Z., Fei, J.: Novel fuzzy neural nonsingular terminal sliding mode control of MEMS gyroscope. Complexity, Article no. 6840639 (2019)

10. Fei, J., Zhou, J.: Robust adaptive control of MEMS triaxial gyroscope using fuzzy compensator. IEEE Transactions on Systems, Man, and Cybernetics: Cybernetics 42(6), 1599-1607 (2012)

11. Vafaie, R., Mohammadzadeh, A., Piran, M.: A new type-3 fuzzy predictive controller for mems gyroscopes. Nonlinear Dynamics 106(1), 381-403 (2021)

12. Zhang, R., Xu, B., Zhao, W.: Finite-time prescribed performance control of MEMS gyroscopes. Nonlinear Dynamics 101(4), 22232234 (2020)

13. Sheng, Y., Bai, W., Xie, Y.: Fractional-order (PID)-D-lambda sliding mode control for hypersonic vehicles with neural network disturbance compensator. Nonlinear Dynamics 103(1), 849-863 (2021)

14. Mehran, H.P., Keighobadi, J.: Robust output regulation of a triaxial MEMS gyroscope via nonlinear active disturbance rejection. International Journal of Robust and Nonlinear Control 28(5), 18301851 (2018)

15. Zheng, Q., Dong, L., Lee, D.H., Gao, Z.: Active disturbance rejection control for MEMS gyroscopes. IEEE Transactions on Control Systems Technology 17(6), 1432-1438 (2009)

16. Fang, Y., Fei, J., Yang, Y.: Adaptive backstepping design of a microgyroscope. Micromachines 9(7), Article no.338 (2018)

17. Mehran, H.P., Jafar, K.: Extended state observer-based robust nonlinear integral dynamic surface control for triaxial MEMS gyroscope. Robotica 37(3), 481-501 (2019)

18. Song, Z., Li, H., Sun, K.: Adaptive dynamic surface control for mems triaxial gyroscope with nonlinear inputs. Nonlinear Dynamics 78(1), 173-182 (2014)

19. Batur, C., Sreeramreddy, T., Khasawneh, Q.: Sliding mode control of a simulated MEMS gyroscope. ISA Transactions 45(1), 99-108 (2006)

20. Fei, J., Batur, C.: Robust adaptive control for a MEMS vibratory gyroscope. International Journal of Advanced Manufacturing Technology 42(3-4), 293-300 (2009)

21. Fei, J., Chen, Y., Liu, L., Fang, Y.: Fuzzy multiple hidden layer recurrent neural control of nonlinear system using terminal sliding-mode controller. IEEE Transactions on Cybernetics DOI 10.1109/TCYB.2021.3052234

22. Rahmani, M., Komijani, H., Ghanbari, A., Ettefagh, M.M.: Optimal novel super-twisting PID sliding mode control of a MEMS gyroscope based on multi-objective bat algorithm. Microsystem Technologies 24(11), 2835-2846 (2018)

23. Fei, J., Liang, X.: Adaptive backstepping fuzzy neural network fractional-order control of microgyroscope using a nonsingular terminal sliding mode controller. Complexity, Article no.5246074 (2018)

24. Xu, B., Zhang, R., Li, S., He, W., Shi, Z.: Composite neural learning-based nonsingular terminal sliding mode control of MEMS gyroscopes. IEEE Transactions on Neural Networks and Learning Systems 31(4), 1375-1386 (2020)

25. Zhang, R., Xu, B., Shi, P.: Output feedback control of micromechanical gyroscopes using neural networks and disturbance observer. IEEE Transactions on Neural Networks and Learning Systems DOI 10.1109/TNNLS.2020.3030712

26. Yang, Q.M., Jagannathan, S., Sun, Y.X.: Robust integral of neural network and error sign control of MIMO nonlinear systems. IEEE Transactions on Neural Networks and Learning Systems 26(12), 3278-3286 (2015)

27. Wang, H., Li, Z., Jin, X., Huang, Y., Kong, H., Yu, M., Ping, Z., Sun, Z.: Adaptive integral terminal sliding mode control for automobile electronic throttle via an uncertainty observer and exper- imental validation. IEEE Transactions on Vehicular Technology 67(9), 8129-8143 (2018)

28. Shao, K., Zheng, J., Huang, K., Wang, H., Man, Z., Fu, M.: Finitetime control of a linear motor positioner using adaptive recursive terminal sliding mode. IEEE Transactions on Industrial Electronics 67(8), 6659-6668 (2020)

29. Hu, Y., Wang, H., He, S., Zheng, J., Ping, Z., Shao, K., Cao, Z., Man, Z.: Adaptive tracking control of an electronic throttle valve based on recursive terminal sliding mode. IEEE Transactions on Vehicular Technology 70(1), 251-262 (2021)

30. Li, S., Ahn, C.K., Xiang, Z.: Command-filter-based adaptive fuzzy finite-time control for switched nonlinear systems using statedependent switching method. IEEE Transactions on Fuzzy Systems 29(4), 833-845 (2021)

31. Zhu, Y., Qiao, J., Guo, L.: Adaptive sliding mode disturbance observer-based composite control with prescribed performance of space manipulators for target capturing. IEEE Transactions on Industrial Electronics 66(3), 1973-1983 (2019) 